\title{
Coma mixedematoso em paciente com neurofibromatose tipo 1: associação rara
}

\author{
Myxedema coma in a patient with type 1 \\ neurofibromatosis: rare association
}

Denise Tieko Sasazawa', Daniela Miti Tsukumo', Cristina Alba Lalli'

\section{SUMÁRIO}

O coma mixedematoso (CM) é uma emergência endocrinológica rara, porém letal e consiste na expressão extrema do hipotireoidismo. Relatamos o caso de um paciente do sexo masculino, 51 anos, que abandonou tratamento do hipotireoidismo por 10 meses e evoluiu com sintomas de letargia, edema e intolerância ao frio que culminaram em insuficiência respiratória e coma. Apresentava também diagnóstico prévio de neurofibromatose. 0 diagnóstico precoce do coma mixedematoso aliado à instituição imediata do tratamento com levotiroxina e ao manejo adequado de complicações, como insuficiência respiratória, choque cardiogênico associado a swinging heart, insuficiências adrenal e renal agudas e sepse, permitiu a evolução favorável do quadro. Arq Bras Endocrinol Metab. 2013;57(9):743-7

\section{SUMMARY}

Myxedema coma, a rare but fatal emergency, is an extreme expression of hypothyroidism. We describe a 51-year-old male patient who has discontinued hypothyroidism treatment 10 months earlier and developed lethargy, edema, and cold intolerance symptoms. He also had a previous diagnosis of neurofibromatosis. After admission, he progressed to respiratory insufficiency and coma. The prompt recognition of the condition, thyroid hormone replacement, and management of the complications (hypoventilation, cardiogenic shock associated with swinging heart, adrenal and renal insufficiency and sepsis), resulted in a favorable evolution. Arq Bras Endocrinol Metab. 2013;57(9):743-7
${ }^{1}$ Departamento de Clínica Médica, Faculdade de Ciências Médicas, Universidade Estadual de Campinas (FCM/Unicamp), Campinas, SP, Brasil

\section{INTRODUÇÃO}

$\mathrm{O}$ coma mixedematoso $(\mathrm{CM})$ é definido como forma extrema de hipotireoidismo, que se instala na falta de tratamento adequado por longo período e em que os mecanismos de compensação são incapazes de manter a homeostase (1). Trata-se de uma emergência endocrinológica rara cuja evolução está intimamente ligada ao diagnóstico precoce e à instituição imediata de seu tratamento. No passado, a mortalidade atingia taxas de até $80 \%$, mas atualmente o avanço na capacidade diagnóstica reduziu esses valores para $20 \%$ a $40 \%(2,3)$.

Acomete principalmente mulheres e idosos, é mais comum no inverno (1) e, geralmente, apresenta um ou mais fatores desencadeantes. Tais fatores incluem exposição ao frio, sepse, drogas (diuréticos, sedativos, analgésicos, anestésicos, amiodarona, lítio, fenitoína), distúrbios hidroeletrolíticos, trauma, acidente vascular cerebral, infarto agudo do miocárdio, insuficiência cardíaca, hemorragias gastrointestinais, queimaduras, anestesia e ingestão de alguns alimentos como o repolho chinês que contém glucosinolatos cujos metabólitos inibem a captação de iodo (1,3-7).

No CM as concentrações séricas de T4 e T3 total e livre estão diminuídas, enquanto o nível de TSH pode estar elevado ou baixo, dependendo se o hipotireoidismo é primário ou secundário.

Em função da presença ubíqua dos receptores tireoidianos, o coma mixedematoso afeta essencialmente todos os órgãos. Sua fisiopatologia inclui diminuição do metabolismo basal e do consumo de oxigênio, vasoconstrição periférica para manutenção da temperatura corporal, diminuição da atividade beta-adrenérgica com manutenção da atividade alfa-adrenérgica que se traduz em hipertensão diastólica, redução do volume sanguíneo, diminuição da frequência e do débito cardíacos $(8,9)$.

A hipotermia, com temperatura corporal menor do que $35^{\circ} \mathrm{C}$, é patognomônica e está presente em $100 \%$ 
dos casos. Em relação ao sistema nervoso central, observam-se sonolência, letargia, desorientação, confusão mental, sintomas psicóticos, depressão e convulsões. O coma pode ser uma manifestação mais tardia (1).

$\mathrm{O}$ aparelho respiratório manifesta-se com insuficiência respiratória grave resultante da diminuição da resposta ventilatória à hipóxia e à hipercapnia, associada à diminuição da força da musculatura torácica e do diafragma, levando a hipoventilação, hipoxemia, hipercapnia, acidose respiratória e narcose. A narcose contribui para a instalação do coma. A obstrução mecânica pela macroglossia e mixedema da orofaringe e a presença de derrame pleural também contribuem para a piora progressiva da ventilação $(1,6,9)$.

Em relação ao sistema cardiovascular, há grave risco de choque e arritmias fatais. A bradicardia sinusal é frequente, enquanto distúrbios de ritmo como Torsades de Pointes e aumento do intervalo QT são menos comuns. Deve-se descartar a concomitância de infarto do miocárdio, uma vez que a terapia com hormônio tireoidiano pode aumentar o risco de evento isquêmico agudo. A contratilidade cardíaca está diminuída e é ainda mais agravada pelo derrame pericárdico. O derrame pericárdico está presente em $25 \%$ dos casos e manifesta-se ao ECG com complexos de baixa voltagem $(6,9)$.

$\mathrm{O}$ trato gastrointestinal apresenta diminuição da motilidade levando à estase gástrica e à constipação intestinal, podendo evoluir a megacólon $(1,9)$.

As alterações do aparelho geniturinário e da função renal incluem retenção urinária, atonia vesical e diminuição da taxa de filtração glomerular. A rabdomiólise com aumento de creatinoquinase também é descrita e contribui para a insuficiência renal. Entre os distúrbios eletrolíticos, o mais frequente é a hiponatremia secundária ao aumento da concentração de $\mathrm{ADH}$ e à menor eliminação de água causada pela redução da água que atinge o néfron distal $(10,11)$. Quando grave, a hiponatremia pode contribuir para a instalação do coma. Também se observam hipertrigliceridemia, hiper ou hipoglicemia, sendo esta última mais frequente $(1,9)$.

Entre as alterações hematológicas, está a anemia, que pode ser microcítica, secundária a sangramento, ou macrocítica por deficiência de vitamina B12. Também podem ocorrer granulocitopenia e deficiência na resposta imunológica celular. Em contraposição ao estado pró-trombótico que ocorre no hipotireoidismo leve, no coma mixedematoso há um alto risco de sangramento secundário à coagulopatia por síndrome de Von Willebrand adquirida e deficiência dos fatores V, VII, IX e X.
Essas alterações são reversíveis com o tratamento com T4 (9).

O tratamento baseia-se na reposição de hormônio tireoidiano, na assistência ventilatória e no manejo das complicações em suporte intensivo e na detecção e tratamento precoce de fatores desencadeantes.

A neurofibromatose tipo 1 (NFl) é doença autossômica dominante. $\mathrm{O}$ gene da NFl foi mapeado e clonado na região pericentromérica do cromossomo $17 q 11.2$ (12). A neurofibromina é um produto do gene que apresenta atividade de uma proteína ativadora da GTPase e é capaz de fazer a regulação negativa da proto-oncogene $\mathrm{p} 2 \mathrm{l}$-ras. A perda de sua função pode levar ao crescimento celular descontrolado e à formação de tumores, daí sua conhecida associação com tumores benignos e malignos. Além disso, a produção anormal de neurofibromina suprime a expressão de fas-ligante, $\mathrm{o}$ que pode evitar a apoptose das células T CD4+, importante no desenvolvimento da autoimunidade (13). Ainda que menos frequente do que sua associação com tumores, a NFl tem sido associada a doenças autoimunes, tais como: esclerose múltipla, esclerose sistêmica, lúpus eritematoso sistêmico, glomerulonefrite membranosa etc. No entanto, há poucos casos relatados de associação de NFl com doença autoimune tireoidiana $(14,15)$.

\section{RELATO DO CASO}

Paciente masculino, 51 anos, com diagnóstico de hipotireoidismo há três anos, foi admitido no inverno de 2010 apresentando quadro de rebaixamento do nível de consciência, dispneia e tosse há um dia. Havia interrompido o tratamento com levotiroxina cerca de 10 meses antes. Nesse período, apresentou os seguintes sintomas, com piora progressiva: adinamia, sonolência, intolerância ao frio, edema e constipação intestinal. Possuía também o diagnóstico prévio de NFl. Ao exame físico geral, apresentava fácies mixedematosa, regular estado geral, mucosas descoradas $(2+/ 4+)$, desidratadas $(3+/ 4+)$, anictéricas e cianóticas $(2+/ 4+)$, pressão arterial $90 / 60 \mathrm{mmHg}$, frequência cardíaca $55 \mathrm{bpm}$, frequência respiratória $24 \mathrm{mpm}$, saturação de $\mathrm{O}_{2} \mathrm{em}$ $86 \%$ em e temperatura de $34^{\circ} \mathrm{C}$ (Figura 1). Apresentava edema frio em membros inferiores, pele fria, espessada, seca, descamativa e amarelada, com manchas ovoides de coloração café com leite e pápulas normocrômicas menores que $1 \mathrm{~cm}$, disseminadas. Também se notavam efélides nas regiões axilares. Apresentava macroglossia e protrusão da língua. A propedêutica da tireoide foi 
prejudicada e não permitiu avaliação da glândula. Ao exame do tórax, apresentava bulhas cardíacas abafadas e ausculta pulmonar com sinais de derrame pleural em bases e estertores crepitantes à direita. $\mathrm{O}$ abdome encontrava-se distendido e com ruídos hidroaéreos diminuídos. O exame neurológico evidenciava coma (escala de Glasgow 9) e reflexos osteotendíneos diminuídos. Avaliação laboratorial inicial na tabela 1. Exames complementares: ECG, bradicardia sinusal e complexos com baixa voltagem em todas as derivações (Figura 2); radiografia do tórax: aumento da área cardíaca, sinais sugestivos de derrame pleural de pequena quantidade bilateral e opacidade homogênea em base direita; ecocardiograma: derrame pericárdico de $16 \mathrm{~mm}$, com repercussão hemodinâmica moderada, padrão denominado swinging heart. A tomografia de crânio e a análise do liquor foram normais. A figura 3 representa a biópsia da pele que confirmou o diagnóstico de NFl.

Foram feitos os diagnósticos de coma mixedematoso desencadeado por pneumonia, insuficiência respiratória e renal agudas e tamponamento cardíaco.

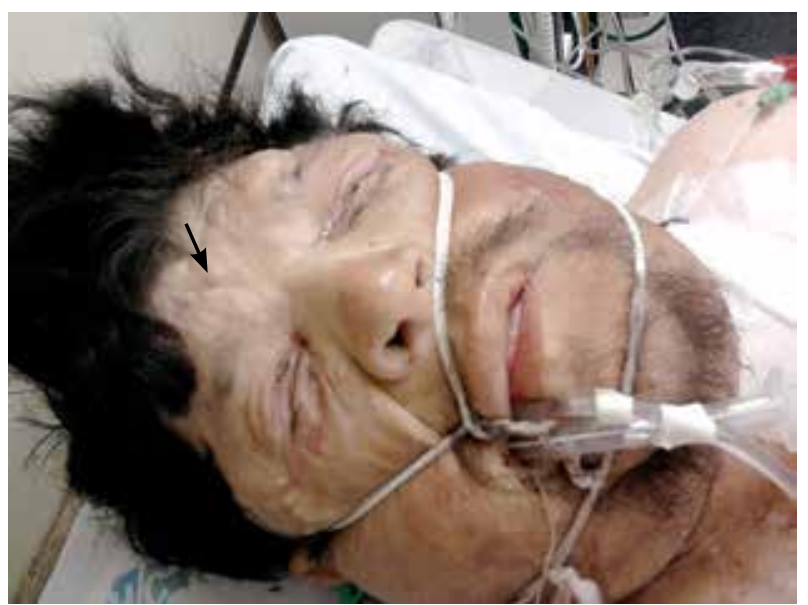

Figura 1. Paciente após intubação orotraqueal: fácies mixedematosa e neurofibromas (seta).

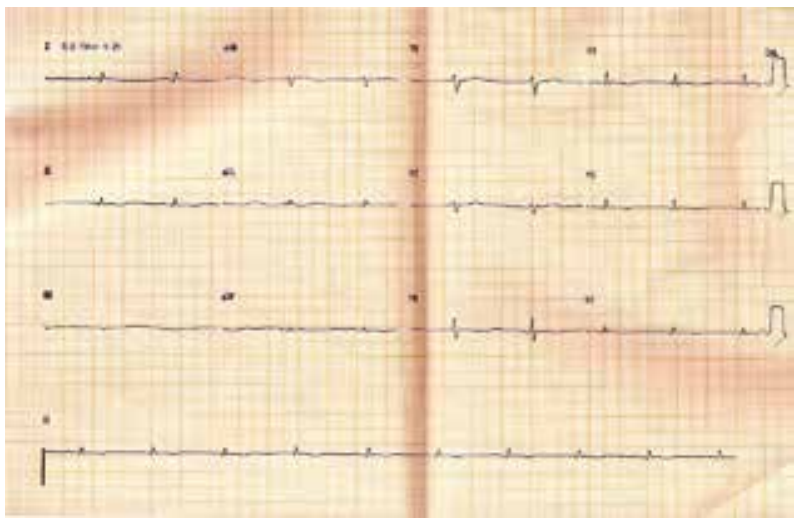

Figura 2. Bradicardia sinusal e complexos de baixa voltagem.

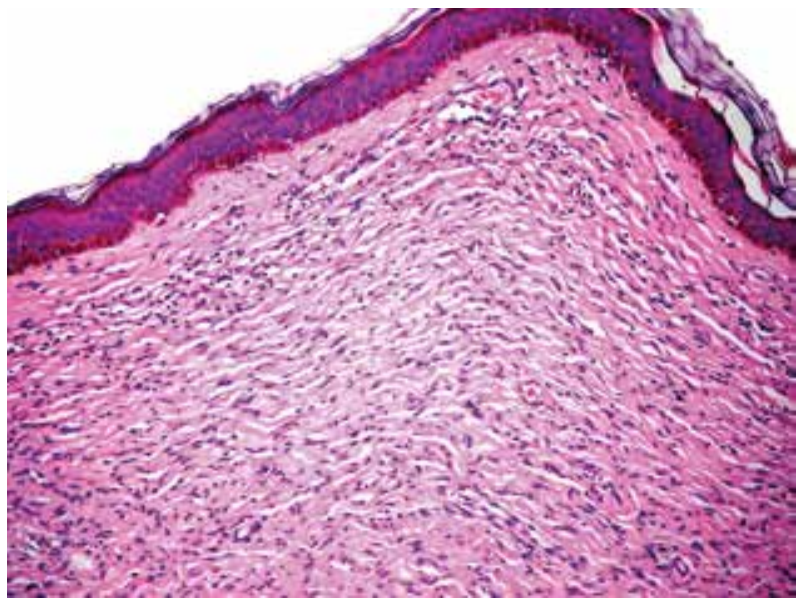

Figura 3. Neurofibroma (hematoxilina eosina): epiderme delgada e derme difusamente espessada por células pequenas, fusiformes, em arranjo frouxo.

Tabela 1. Exames laboratoriais

\begin{tabular}{|c|c|}
\hline Exame e valor de referência & Resultado \\
\hline TSH $(0,41-4,5 \mu \mathrm{U} / \mathrm{mL})$ & $>100$ \\
\hline T4I (0,9 - 1,8 $\mu \mathrm{U} / \mathrm{mL})$ & 0,07 \\
\hline T3I (0,257 - 0,443 ng/dL) & 0,1 \\
\hline Leucócitos totais (4,000 a 10,000/mm³) & 1,000 \\
\hline Bastões $\left(0-700 / \mathrm{mm}^{3}\right)$ & 240 \\
\hline Segmentados $\left(20,000-80,000 / \mathrm{mm}^{3}\right)$ & 620 \\
\hline Linfócitos $\left(1,000-40,000 / \mathrm{mm}^{3}\right)$ & 120 \\
\hline Hemoglobina (14-18 g/dL) & 6,8 \\
\hline Hematócrito (42-52\%) & 25,7 \\
\hline Plaquetas $\left(150,000-400,000 \mathrm{l} / \mathrm{mm}^{3}\right)$ & 24,000 \\
\hline Glicemia (70-99 mg/dL) & 74 \\
\hline Creatinina (<1,2 mg/dL) & 1,6 \\
\hline Ureia $(<50$ mg/dL) & 76 \\
\hline Sódio (136-145 mEq/l) & 141 \\
\hline Potássio (3,1-5,1 mEq/l) & 3,8 \\
\hline Creatinoquinase (< 170 U/I) & 748 \\
\hline Troponina $(>0,01 \mathrm{ng} / \mathrm{dl} \mathrm{e}<0,1 \mathrm{ng} / \mathrm{dL}$ ) & 0,09 \\
\hline Aspartato aminotransferase (< $50 \mathrm{U} / \mathrm{l})$ & 32 \\
\hline Alanino aminotransferase (<33 U/l) & 12 \\
\hline Bilirrubina total (<1,0 mg/dL) & 0,66 \\
\hline Anticorpo antitireoglobulina (< $155 \mathrm{Ul} / \mathrm{mL})$ & 988,40 \\
\hline Anticorpo antiperoxidase (< $35 \mathrm{UI} / \mathrm{mL})$ & Negativo \\
\hline
\end{tabular}

Imediatamente foram iniciadas reposição volêmica e entubação orotraqueal para ventilação mecânica, administração de droga vasoativa (noradrenalina) e de hidrocortisona $100 \mathrm{mg}$ de $6 / 6$ horas e reposição com levotiroxina sódica $500 \mu \mathrm{g}$ via sonda naso-enteral. Iniciada terapia com antibiótico de largo espectro e rea- 
lizada drenagem pericárdica com saída de $350 \mathrm{~mL}$ de líquido de coloração citrina, sem outras anormalidades. A dose de manutenção de T4 foi de $200 \mu$ g ao dia. A função renal deteriorou progressivamente e foi necessária terapia renal substitutiva. A internação prolongou-se, com várias complicações e infecções nosocomiais, mas o paciente recebeu alta hospitalar após 50 dias para seguimento ambulatorial, com prescrição de levotiroxina $175 \mu \mathrm{g}$ ao dia.

\section{DISCUSSÃO}

Desde a publicação do primeiro caso de CM em 1879, relativamente poucos casos foram reportados na literatura até os dias de hoje e, em decorrência de sua raridade, poucos estudos clínicos estão disponíveis para a determinação de critérios diagnósticos, do tratamento e da avaliação de desfechos $(1,8)$. Os critérios são inespecíficos, não existem marcadores laboratoriais adequados e os níveis de TSH e T4 livre não se relacionam diretamente com a gravidade do caso. Em contrapartida, observa-se aumento da prevalência do hipotireoidismo devido aos avanços no seu diagnóstico e ao aumento da prática de radioiodoterapia e tireoidectomia como formas de tratamento definitivo do hipertiroidismo $(1,3,4,8)$.

A maior controvérsia no manejo do CM é qual o hormônio tireoidiano escolher, T4 ou T3, em qual dose, apresentação e via de administração, devendo-se contrabalançar a necessidade da rápida reposição das necessidades fisiológicas com o risco de arritmias fatais e infarto do miocárdio (9).

Vários esquemas terapêuticos têm sido propostos. A administração de dose inicial de T4 por via endovenosa na dose de 300-600 $\mu \mathrm{g}$ seria suficiente para suprir o grande número de sítios de ligação livres das globulinas transportadoras e permitiria rapidez na reconstituição dos níveis circulantes de T4. Esse esquema seguiria com dose de manutenção de 50 a $100 \mu$ g por dia (9).

A conversão de T4 a T3 está diminuída em várias doenças sistêmicas graves (síndrome do eutireoidiano doente) e, no CM, a presença de doenças associadas diminuiria ainda mais o T3 disponível e poderia justificar seu uso no tratamento. São sugeridos esquemas com T3 isolado ou associado a T4. No tratamento com T3, é observado efeito sobre a temperatura e o consumo de $\mathrm{O}_{2}$ em poucas horas. No entanto, alguns trabalhos mostraram que o uso de T3 está relacionado a maior risco de arritmias, infarto do miocárdio e aumento de mortalidade $(1,9,16)$.
As preparações parenterais não estão disponíveis em nosso meio, e a administração por via oral ou por sonda nasogástrica tem a desvantagem da absorção errática, principalmente na presença de atonia gástrica e íleo paralítico, e do risco de aspiração. Em nosso caso, a dose de manutenção utilizada foi mais alta do que a preconizada, pois a administração por sonda de fato resultou em absorção mais lenta e demora em atingir níveis adequados de T4.

Enfatiza-se, com este relato, a importância do diagnóstico precoce do $\mathrm{CM}$, aliado à instituição de seu tratamento com levotiroxina prontamente e ao manejo adequado do espectro de complicações clínico-cirúrgicas e da busca ativa dos fatores precipitantes. Também evidenciamos a rara associação de tireoidite autoimune com NFl.

Agradecimentos: não houve apoio financeiro ao presente trabalho.

Declaração: os autores declaram não haver conflitos de interesse científico neste estudo.

\section{REFERÊNCIAS}

1. Kwaku MP, Burman KD. Myxedema coma. J Intensive Care Med. 2007;22:224-31.

2. Wartofsky L. Myxedema coma. In: The Thyroid: A Fundamental and Clinical Text, Braverman LE, Utiger RD (eds.). Philadelphia Lippincott: Williams \& Wilkins; 2000. p. 843.

3. Dutta P, Bhansali A, Masoodi S, Bhadada S, Sharma N, Rajput R. Predictors of outcome in myxedema coma: a study from a tertiary care centre. Critical Care. 2008;12:S1.

4. Rodríguez I, Fluiters E, Pérez-Méndez LF, Luna R, Páramo C, García-Mayor RV. Factors associated with mortality of patients with myxoedema coma: prospective study in 11 cases treated in a single institution. J Endocrinol. 2004;180:347-50.

5. KearneyT, Dang C. Diabetic and endocrine emergencies. Postgrad Med J. 2007;83:79-86.

6. Danilovic DLS, Almeida MQ, Neto RAB, Martins H. Coma Mixedematoso. In: Emergências Clinicas Abordagem Prática 7th Ed. Barueri: Manole; 2012. p. 974-80.

7. Santiago R, Rashkin MC. Lithium toxicity and myxedema coma in an elderly woman. J Emerg Med. 1990;8:63.

8. Park CW, Shin YS, Ahn SJ, Kim SY, Choi EJ, Chang YS, et al. Thyroxine treatment induces upregulation of renin-angiotensinaldosterone system due to decreasing effective plasma volume in patients with primary myxoedema. Nephrol Dial Transplant. 2001;6:1799-806.

9. Klubo-Gwieedzinska J, Wartofsky L. Thyroid emergencies. Med Clin N Am. 2012;96:385-403.

10. Villabona C, Sahun M, Roca M, Mora J, Gomez N, Gomez JM, et al. Blood volumes and renal function in overt and subclinical primary hypothyroidism. Am J Med Sci. 1999;318:277-80.

11. Iwasaki $\mathrm{Y}$, Oiso $\mathrm{Y}$, Yamauchi $\mathrm{K}$, et al. Osmoregulation of plasma vasopressin in myxedema. J Clin Endocrinol Metab. 1990;70:534-9.

12. Barker D, Wright E, Nguyen K, Cannon L, Fain P, Goldgar $D$, et al. Gene for von Recklinghausen neurofibromatosis is in the pericentromeric region of chromosome 17. Science. 1987;236:1100-2. 
13. Abbas A, Lichtman AH. Disease caused by immune responses: hipersensitivity and autoimmunity. In: Abbas $A$, Lichtman $A H$, eds. Cellular and molecular immunology 5 th ed. Philadelphia: Saunders; 2005. p. 411-31.

14. Yalcin B, Tamer E, Gür G, Oztas P, Polat MU, Alli N. Neurofibromatosis 1/Noonan syndrome associated with Hashimoto's thyroiditis and vitiligo. Acta Derm Venereol. 2006;86:80-1.
15. Nanda A. Autoimmune diseases associated with neurofibromatosis type 1. Ped Dermatology. 2008;25(3):392-3.

16. MacKerrow SD, Osborn LA, Levy $H$, Eaton RP, Economou P. Myxedema-associated cardiogenic shock treated with intravenous triiodothyronine. Ann Intern Med. 1992;117:1014-5. 